\title{
Rotating flow in a cylinder with a circular barrier on the bottom
}

\author{
G. J.F. van HEIJST \\ Department of Technical Physics, Twente University of Technology, Enschede, \\ The Netherlands.
}

(Received July 28, 1978)

\section{SUMMARY}

The relative flow of a homogeneous, slightly viscous fluid in a rotating cylinder is induced by differential rotation of the bottom disk, on which a thin circular strip of small height is fixed. The axis of symmetry of the strip coincides with the rotation axis of the cylinder.

At the strip a Stewartson layer exists which is partially free, partially attached to the strip. The structure of the Stewartson $E^{1 / 4}$-layer ( $E$ being the Ekman number) is not affected by the height of the strip, but the $E^{1 / 3}$-layer problem has to be solved in the two separate intervals. The fact that both solutions do not match at the strip edge necessitates the presence of an intermediate region that exhibits some characteristic features of an Ekman layer.

\section{Introduction}

In recent years, partly stimulated by applications in oceanography and meteorology, there has been considerable interest in relative flows in a rotating system driven by the differential rotation of some cylinder part. At sufficiently small values of the differential rotation the flow is one of almost rigid rotation and can in a number of situations be studied analy tically. A central phenomenon is the axial shear layer, first studied by Stewartson [7], which possesses a complex multi-layered structure. Such shear layers may be free, as in the work of Stewartson [7,8] and Moore \& Saffman [6], or attached to some solid boundary. Foster [2,3] has considered the structure of a Stewartson layer that is partially free, partially attached to a solid boundary. In Foster [2] the Stewartson layer was induced by the differential rotation of a circular cylindrical depression in one of two rotating parallel planes. In many practical situations obstacles occur on one or both of the horizontal disks. Boyer [1] has studied bottom topographies where these obstacles have lateral dimensions of the order of magnitude of the cylinder radius. In technical situations often one of these dimensions is small: barriers or strips on the bottom of centrifuges or mixers.

In a program set up to investigate the effect of such strips or barriers we found that a number of new and complicated problems arise, in particular when the flow looses axial symmetry. In order to analyse one of these problems, viz. a shear layer which is partly free, partly 
attached to a strip, while keeping the flow axially symmetric, we consider the situation shown in Figure 1.

An incompressible, slightly viscous fluid is contained in a right circular cylinder rotating about its (vertical) axis, while the bottom plate of the cylinder rotates with a slightly different angular velocity. $L$ and $H L$ denote radius resp. height of the cylinder. On the bottom disk a thin circular strip with radius $a L$ and height $h L$ is fixed, its axis of symmetry coinciding with the rotation axis of the entire system. The thickness $\delta$ of the strip is assumed to be small $(\delta<<h)$. In the following analysis we shall use non-dimensional cylindrical polar coordinates $(r, \theta, z)$, with corresponding velocity components $(u, v, w)$. We confine ourselves to the case that the bottom rotates at speed $\Omega$, while the remainder of the cylinder has angular velocity $\Omega(1+\epsilon)$ with $0<\epsilon<<1$. The pertinent Rossby number of the flow is $\epsilon$, and for sufficiently small $\epsilon$ nonlinear effects can be neglected. Further we consider the flow in the limit $E \rightarrow 0$.

The governing equations with the general boundary conditions for the flow problem are formulated in Sec. 2. In Sec. 3 we present the solutions for the fluid motion in the interior regions $I_{1}(0<r<a, 0<z<H)$ and $I_{2}(a<r<1,0<z<H)$ in which the flow is geostrophic. As usual, Ekman layers occur on both top and bottom plane, which are also dealt with in Sec. 3 . On the vertical cylinder wall $(r=1)$ a Stewartson layer is present, the analysis of which is straight forward, and will be omitted here. Another Stewartson layer exists near the cylindrical plane $r=a, 0<z<H$ which forms the boundary between both interior domains $I_{1}$ and $I_{2}$. As will be discussed in Sec. 4, this shear layer exhibits some interesting new features, because for $h<z<H$ it is free, while for $0<z<h$ the layer is attached to the solid strip boundaries $r=a^{-}$and $r=a^{+}$(see Figure 2). In this sense our problem is related to the study of Foster [2]. The difference lies in the fact that the attached shear layer consists of two separate parts, on both strip surfaces $r=a^{-}$and $r=a^{+}$. For this reason the coupling of both types of Stewartson layers at $z=h$ differs from Foster's problem. The outer part of the layer, with typical thickness $E^{1 / 4}$, will prove to be independent of the strip height $h$, in contrast with the inner part, of typical thickness $E^{1 / 3}$. In order to carry a mass flux this $1 / 3$-layer has to possess a singularity

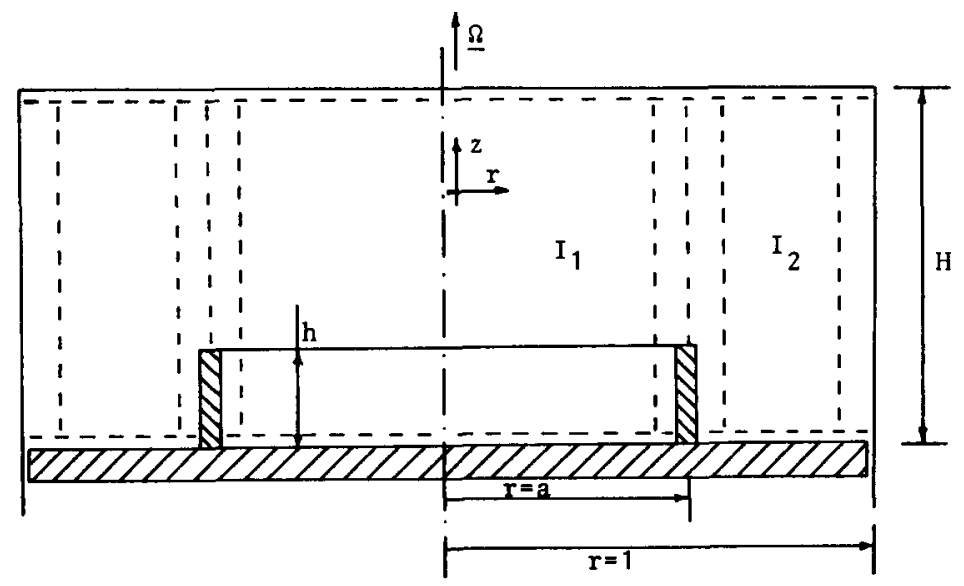

Figure 1. Geometrical configuration. Lengths have been made dimensionless with the radius $L$ of the cylinder; shear layers are denoted by broken lines. $I_{1}(0<r<a, 0<z<H)$ and $I_{2}(a<r<1,0<z<H)$ are both interior regions, in which the flow is geostrophic. 


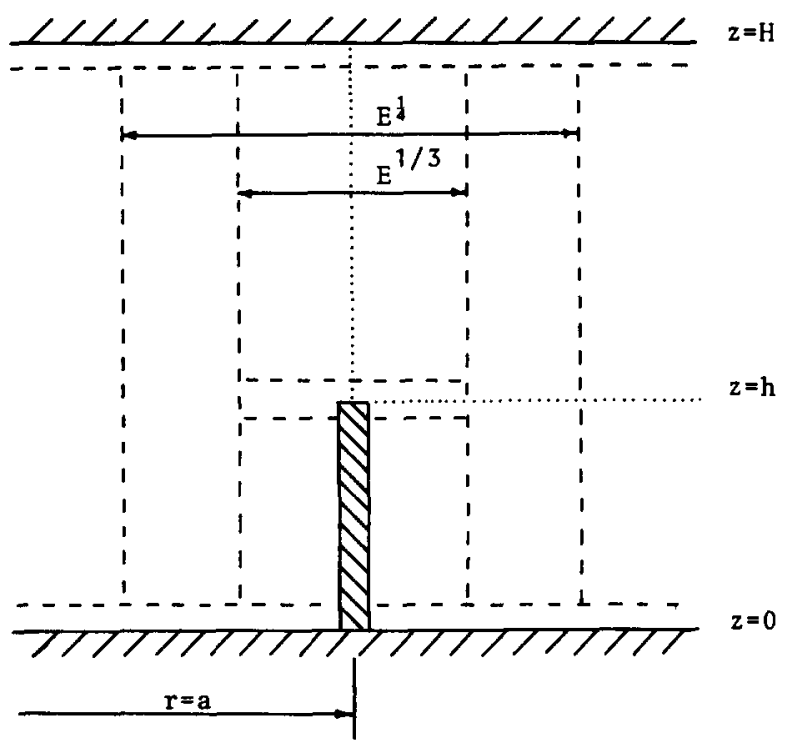

Figure 2. Structure of the Stewartson layer at $r=a$.

at the strip edge $r=a, z=h$. Since the 1/3-layer parts in $0<z<h$ and $h<z<H$ do not match smoothly at $z=h$, an intermediate region at $h^{-}<z<h^{+}$will be necessary, which will be discussed in Sec. 5. This intermediate regions forms a new feature in rotating boundary layer theory. Because of its strong analogy to the usual Ekman layer, it is called here 'quasi-Ekman layer'.

\section{Formulation of the problem}

Relative to a uniformly rotating frame of reference, the equations governing the motion of an incompressible homogeneous fluid of density $\rho$ and kinematic viscosity $\nu$, are (see e.g. Greenspan [4]):

$$
\begin{gathered}
\frac{\partial \mathrm{q}^{*}}{\partial t^{*}}+\left(\mathrm{q}^{*} \cdot \nabla^{*}\right) \mathrm{q}^{*}+2 \Omega \times \mathrm{q}^{*}=-\frac{1}{\rho} \nabla^{*} p^{*}+\nu \nabla^{* 2} \mathrm{q}^{*}, \\
\nabla^{*} \cdot \mathrm{q}^{*}=0,
\end{gathered}
$$

where $\mathrm{q}^{*}, p^{*}, t^{*}, \Omega$ respectively represent velocity, pressure, time, and the rotation vector of the system.

Let $L, \Omega^{-1}, U$ denote the typical length, time and relative velocity of the fluid motion so that the governing equations can be written in a nondimensional form:

$$
\begin{gathered}
\frac{\partial \mathbf{q}}{\partial t}+R o(\mathbf{q} \cdot \nabla) \mathbf{q}+2 \mathbf{k} \times \mathbf{q}=-\nabla p+E \nabla^{2} \mathbf{q}, \\
\nabla \cdot \mathbf{q}=0,
\end{gathered}
$$

* In fact, a similar region is considered in unpublished work by J. A. Johnson (private communication) 
where $\mathbf{k}=\Omega / \Omega$ is the unit vector of rotation. Two dimensionless variables appear: the Rossby number $R o=U / \Omega L$, and the Ekman number $E=\nu / \Omega L^{2}$. In steady axisymmetric flow with small Rossby number the velocity components $(u, v, w)$ in a cylindrical polar coordinate system $(r, \theta, z)$ obey, from (2.2) and (2.3), the equations

$$
\begin{gathered}
-2 v=-\frac{\partial p}{\partial r}+E\left(\nabla^{2} u-\frac{u}{r^{2}}\right) \\
2 u=\quad E\left(\nabla^{2} v-\frac{v}{r^{2}}\right) \\
0=-\frac{\partial p}{\partial z}+E \nabla^{2} w \\
\frac{1}{r} \frac{\partial}{\partial r}(r u)+\frac{\partial w}{\partial z}=0
\end{gathered}
$$

with

$$
\nabla^{2}=\frac{\partial^{2}}{\partial r^{2}}+\frac{1}{r} \frac{\partial}{\partial r}+\frac{\partial^{2}}{\partial z^{2}}
$$

The boundary conditions for (2.4) can be formulated (see Figure 1) as:

$$
\begin{aligned}
& z=0,0<r<1: q=(0,0,0), \\
& z=H, 0<r<1: q=(0, r, 0) ; \\
& r=1,0<z<H: q=(0,1,0), \\
& r=a, 0<z<h: q=(0,0,0) .
\end{aligned}
$$

\section{The interior solution and the Ekman layers}

In the interior, that is outside boundary layers of Stewartson or Ekman type, the velocity components and pressure may be expanded in the following perturbation series

$$
\begin{aligned}
& u=u_{I}^{(0)}+E^{\frac{1}{4}} u_{I}^{(1)}+E^{\frac{1}{2}} u_{I}^{(2)}+\ldots \ldots, \\
& v=v_{I}^{(0)}+E^{\frac{1}{4}} v_{I}^{(1)}+E^{\frac{1}{2}} v_{I}^{(2)}+\ldots \ldots \\
& w=E^{\frac{1}{2}} w_{I}^{(0)}+E^{\frac{3}{4}} w_{I}^{(1)}+E w_{I}^{(2)}+\ldots \ldots, \\
& p=p_{I}^{(0)}+E^{\frac{1}{4}} p_{I}^{(1)}+E^{\frac{1}{2}} p_{I}^{(2)}+\ldots \ldots
\end{aligned}
$$

where the index $I$ denotes 'interior'. Substitution of these expansions and letting $E \rightarrow 0$ reduces (2.4) to 


$$
\begin{aligned}
-2 v_{I}^{(i)}=-\frac{\partial}{\partial r} p_{I}^{(i)}, & u_{I}^{(i)}=0, \frac{\partial}{\partial z} p_{I}^{(i)}=0, \\
& \frac{1}{r} \frac{\partial}{\partial r}\left(r u_{I}^{(i)}\right)+E^{\frac{1}{2}} \frac{\partial w_{I}^{(i)}}{\partial z}=0, \text { for } i=0,1,2 .
\end{aligned}
$$

From these equations we deduce

$$
\frac{\partial}{\partial z} \mathbf{v}_{I}^{(i)}=\frac{\partial}{\partial z} p_{I}^{(i)}=0
$$

known as the 'Taylor-Proudman'-theorem.

The Ekman layers at $z=0, z=H$ may be analysed in the usual way with $u_{E}, \nu_{E}, p_{E}$ of $O(1)$ and $w_{E}$ of $O\left(E^{\frac{1}{2}}\right)$, obeying

$$
\begin{aligned}
-2 v_{E} & =-\frac{\partial p_{E}}{\partial r}+E \frac{\partial^{2} u_{E}}{\partial z^{2}}, \\
2 u_{E} & =\quad E \frac{\partial^{2} v_{E}}{\partial z^{2}}, \\
0 & =-\frac{\partial p_{E}}{\partial z}, \\
\frac{1}{r} & \frac{\partial}{\partial r}\left(r u_{E}\right)+E^{\frac{1}{2}} \frac{\partial w_{E}}{\partial z}=0 .
\end{aligned}
$$

These equations provide as constraint on the adjacent flow, the Ekman suction condition

$$
w_{\Sigma}=\frac{(\mathbf{n} \cdot \mathbf{k})}{2 r} E^{\frac{1}{2}} \frac{\partial}{\partial r}\left\{r\left(v_{\Sigma}-V\right)\right\},
$$

where $\mathbf{n}$ is the outward normal to the concerning rigid wall, and $V$ denotes the swirl velocity of this boundary. The velocities in the adjacent flow region are denoted by the suffix $\Sigma$. Since in our case $V(z=0)=0$ and $V(z=H)=r$, the Ekman suction conditions for the interior flow become:

$$
w_{I}^{(i)}= \pm \frac{1}{2 r} \frac{\partial}{\partial r}\left\{r\left(v_{I}^{(i)}-V\right)\right\} \quad \text { for } z=(1 \mp 1) \frac{H}{2} .
$$

The solution for the interior motion, subject to the conditions (2.5b) for $r=1$, and (3.6), is to leading order

$$
u_{I}^{(0)}=0, \quad v_{I}^{(0)}=\frac{1}{2} r, \quad w_{I}^{(0)}=\frac{1}{2} .
$$

According to this solution the interior motion is a rigid-body rotation with angular velocity equal to the arithmetric average of both top and bottom plane rotation rates. Besides, a weak 
axial motion of $O\left(E^{\frac{1}{2}}\right)$ is induced by the differential Ekman suction, directed towards the faster rotating disk. It can be seen from (3.7) that the tangential velocity cannot be zero for $r=a$, $0<z<h$ (due to the no-slip condition on the strip surface), and because of the Taylor-Proudman theorem (3.3) we must conclude that a vertical shear layer is necessary to bring $v_{I}{ }^{(0)}$ to zero at $r=a$. Solving the equations (3.4) yields for lower resp. upper Ekman layer

$$
\left.\begin{array}{l}
\left.\begin{array}{l}
u_{E}=-\frac{1}{2} r e^{-\zeta} \sin \zeta \\
v_{E}=\frac{1}{2} r\left\{1-e^{-\zeta} \cos \zeta\right\}
\end{array}\right\} \text { with } \zeta=z E^{-\frac{1}{2}}, \\
u_{E}=\frac{1}{2} r e^{-\zeta} \sin \zeta \\
v_{E}=\frac{1}{2} r\left\{1+e^{-\zeta} \cos \zeta\right\}
\end{array}\right\} \text { with } \zeta=(H-z) E^{-\frac{1}{2}},
$$

from which may be calculated the radial Ekman transports

$$
Q_{\mathrm{rad}}(z=0)=-\frac{r}{4} E^{\frac{1}{2}}, \quad Q_{\mathrm{rad}}(z=H)=+\frac{r}{4} E^{\frac{1}{2}},
$$

expressing the phenomenon that the faster rotating disk acts as a centrifugal fan.

\section{The Stewartson layer}

As stated in Sec. 3, the interior tangential velocity $v_{I}$ must be matched to zero on the cylindrical plane $r=a$. This can be accomplished by two 1/4-layers at $r=a^{-}, a^{+}$which run, independent of the height of the strip, from $z=0$ to $z=H$. It will become clear that a thinner $1 / 3$-layer will be needed. The $1 / 3$-layer equations, however, must be solved separately in the regions $0<z<h$ and $h<z<H$. Since these solutions do not match smoothly at $z=h$, an "intermediate region" is needed. This domain possesses some characteristic features of the usual Ekman layer, and is therefore called here "quasi-Ekman layer".

\section{a. the 1/4-layer}

For this shear layer we expand $q$ and $p$ into the following perturbation series:

$$
\begin{aligned}
& u(r, z ; E)=E^{\frac{1}{4}} U^{(0)}(\xi, z)+E^{\frac{1}{2}} U^{(1)}(\xi, z)+\ldots, \\
& v(r, z ; E)=V^{(0)}(\xi, z)+E^{\frac{1}{4}} V^{(1)}(\xi, z)+\ldots, \\
& w(r, z ; E)=E^{\frac{1}{4}} W^{(0)}(\xi, z)+E^{\frac{1}{2}} W^{(1)}(\xi, z)+\ldots \\
& p(r, z ; E)=E^{\frac{1}{4}} P^{(0)}(\xi, z)+E^{\frac{1}{2}} P^{(1)}(\xi, z)+\ldots,
\end{aligned}
$$

where $\xi=(r-a) E^{-\frac{1}{4}}$.

Substitution of these expansions in the basic equations (2.4) leads to 


$$
\begin{aligned}
& 2 V^{(0)}=\frac{\partial P^{(0)}}{\partial \xi}, 2 V^{(1)}=\frac{\partial P^{(1)}}{\partial \xi}, 2 V^{(2)}=\frac{\partial P^{(2)}}{\partial \xi}, \\
& U^{(0)}=0,2 U^{(1)}=\frac{\partial^{2} V^{(0)}}{\partial \xi^{2}}, 2 U^{(2)}=\frac{\partial^{2} V^{(1)}}{\partial \xi^{2}}+\frac{1}{a} \frac{\partial V^{(0)}}{\partial \xi}, \\
& \frac{\partial P^{(0)}}{\partial z}=0, \frac{\partial P^{(1)}}{\partial z}=0, \frac{\partial P^{(2)}}{\partial z}=\frac{\partial^{2} W^{(0)}}{\partial \xi^{2}}, \\
& \frac{\partial U^{(0)}}{\partial \xi}=0, \frac{\partial U^{(1)}}{\partial \xi}+\frac{1}{a} U^{(0)}+\frac{\partial W^{(0)}}{\partial z}=0, \frac{1}{a} U^{(1)}-\frac{\xi}{a^{2}} U^{(0)}+\frac{\partial U^{(2)}}{\partial \xi}+\frac{\partial W^{(1)}}{\partial z}=0,
\end{aligned}
$$

while the Ekman suction conditions (3.5) can be written as

$$
\begin{aligned}
& W^{(0)}= \pm \frac{1}{2} \frac{\partial V^{(0)}}{\partial \xi}, \\
& W^{(1)}= \pm \frac{1}{2}\left\{\frac{\partial V^{(0)}}{\partial \xi}+\frac{1}{a} V^{(0)}\right\}+\left(\frac{1}{2} \mp \frac{1}{2}\right), \\
& W^{(2)}= \pm \frac{1}{2}\left\{\frac{\partial V^{(2)}}{\partial \xi}+\frac{1}{a} V^{(1)}-\frac{\xi}{a^{2}} V^{(0)}\right\},
\end{aligned}
$$

at $z=\frac{1}{2}(1 \mp 1) H$.

For $\xi \rightarrow \pm \infty$ the 1/4-layer solutions have to match the interior solution (3.7), while at $\xi=0$ matching to the thinner 1/3-layer is demanded. For the $\left(U^{(1)}, V^{(0)}, W^{(0)}\right)$ velocity field we deduce from $(4.2 \mathrm{a})-(4.2 \mathrm{~d})$

$$
\frac{\partial^{3} V^{(0)}}{\partial \xi^{3}}-\frac{2}{H} \frac{\partial V^{(0)}}{\partial \xi}=0
$$

the solutions of which are

$$
\begin{aligned}
& V_{+}^{(0)}(\xi)=C_{1}^{+} \exp \left(-\xi \sqrt{\frac{2}{H}}\right)+C_{2}^{+} \quad(\xi \geqslant 0), \\
& V_{-}^{(0)}(\xi)=C_{1}^{-} \exp \left(+\xi \sqrt{\frac{2}{H}}\right)+C_{2}^{-} \quad(\xi \leqslant 0) .
\end{aligned}
$$

Applying the conditions at $\xi \rightarrow \pm \infty$ we obtain $C_{2}^{+}=C_{2}^{-}=\frac{1}{2} a$. The constants $C_{1}^{ \pm}$are to be determined using the equations for the 1/3-layer interior to the two 1/4-layers, as Stewartson [8] has pointed out. The way in which this carried out, depends on the geometry of the concerning flow problem. In the split-disk problem of Stewartson [7] the conditions for determining $C_{1}^{ \pm}$are that $V$ and $\partial V /(\partial \xi)$ should be continuous at $\xi=0$. The jump conditions for the $1 / 4$-layers in the rising disk problem of Moore \& Saffman [6] are $V$ and $\partial^{2} V /\left(\partial \xi^{2}\right)$ being continuous on $\xi=0$, implicating that $\partial V /(\partial, \xi)$ is discontinuous at $\xi=0$. 
It can be verified that in the present case the jump conditions take this latter form, and after using the appropriate Ekman suction conditions (4.3), one obtains

$$
\begin{aligned}
& V_{+}^{(0)}(\xi)=\frac{1}{2} a\left\{1-\exp \left(-\xi \sqrt{\frac{2}{H}}\right)\right\} \\
& V_{-}^{(0)}(\xi)=\frac{1}{2} a\left\{1-\exp \left(+\xi \sqrt{\frac{2}{H}}\right)\right\} \\
& W_{+}^{(0)}(\xi, z)=-\frac{1}{2} a \sqrt{\frac{2}{H}}\left(\frac{z}{H}-\frac{1}{2}\right) \exp \left(-\xi \sqrt{\frac{2}{H}}\right) \\
& W_{-}^{(0)}(\xi, z)=+\frac{1}{2} a \sqrt{\frac{2}{H}}\left(\frac{z}{H}-\frac{1}{2}\right) \exp \left(+\xi \sqrt{\frac{2}{H}}\right)
\end{aligned}
$$

We note that

- $\quad V^{(0)}$ and $\partial^{2} V^{(0)} /\left(\partial \xi^{2}\right)$ are continuous at $\xi=0$,

- $\partial V^{(0)} /(\partial \xi)$ is discontinuous at $\xi=0$.

- $W^{(0)}$ is neither continuous at $\xi=0$, nor does it satisfy the no-slip condition $W^{(0)}(\xi=0)=0$ at $0<z<h$,

- the vertical $O\left(E^{\frac{1}{2}}\right)$-transports in the 1/4-layers,

$$
\begin{gathered}
E^{\frac{1}{2}} \int_{-\infty}^{0^{-}} W_{-}^{(0)}(\xi, z) d \xi=+\frac{1}{2} a\left(\frac{z}{H}-\frac{1}{2}\right) E^{\frac{1}{2}}, \\
E^{\frac{1}{2}} \int_{0^{+}}^{+\infty} W_{+}^{(0)}(\xi, z) d \xi=-\frac{1}{2} a\left(\frac{z}{H}-\frac{1}{2}\right) E^{\frac{1}{2}},
\end{gathered}
$$

are balanced by the local Ekman transports (3.9) at $z=0$ and $z=H$ for $r=a$. The net vertical $O\left(E^{\frac{1}{2}}\right)$-transport in the 1/4-layer appears to be zero.

In the $1 / 3$-layer analysis we shall use these properties of the $1 / 4$-layer, and derive conditions for the 1/3-layer from it. In the analysis of the 1/3-layer later in this section, we shall need also the velocity $V^{(1)}$ in the $1 / 4$-layer, which has to satisfy, from $(4.2 \mathrm{a})-(4.2 \mathrm{~d})$, the equation

$$
\frac{\partial^{3} V^{(1)}}{\partial \xi^{3}}-\frac{2}{H} \frac{\partial V^{(1)}}{\partial \xi}=-\frac{2}{a} \frac{\partial^{2} V^{(0)}}{\partial \xi^{2}}+\frac{2}{a H} V^{(0)}-\frac{2}{H} .
$$

This equation possesses the following solutions with exponential decay for large $|\xi|$ :

$$
\begin{aligned}
& V_{+}^{(1)}(\xi)=D_{1}^{+}+\frac{1}{2} \xi+D_{2}^{+} \exp \left(-\xi \sqrt{\frac{2}{H}}\right)+\frac{1}{4} \xi \exp \left(-\xi \sqrt{\frac{2}{H}}\right) \\
& V_{-}^{(1)}(\xi)=D_{1}^{-}+\frac{1}{2} \xi+D_{2}^{-} \exp \left(+\xi \sqrt{\frac{2}{H}}\right)-\frac{1}{4} \xi \exp \left(+\xi \sqrt{\frac{2}{H}}\right)
\end{aligned}
$$

where $D_{1}^{ \pm}$and $D_{2}^{ \pm}$are integration constants, to be determined from matching to the interior $(\xi \rightarrow \pm \infty)$ and the inner 1/3-layer $(\xi \rightarrow \pm 0)$.

From (4.8) follows: 


$$
\begin{aligned}
& V_{+}^{(1)}(\xi=0)=D_{1}^{+}+D_{2}^{+}=\text {constant } \\
& V_{-}^{(1)}(\xi=0)=D_{1}^{-}+D_{2}^{+}=\text {constant }
\end{aligned}
$$

We shall use this result in the discussion of the 1/3-layer.

\section{b. the 1/3-layer}

In this inner layer the velocity components and the pressure are expanded in powers of $E^{1 / 12}$ :

$$
\begin{aligned}
& u=E^{4 / 12} \tilde{u}^{(0)}+E^{5 / 12} \tilde{u}^{(1)}+E^{6 / 12} \tilde{u}^{(2)}+E^{7 / 12} \tilde{u}^{(3)}+\ldots . \\
& \nu=\mathfrak{\vartheta}^{(0)}+E^{1 / 12} \mathcal{\nu}^{(1)}+E^{2 / 12 \mathcal{\nu}^{(2)}}+E^{3 / 12} \mathcal{\nu}^{(3)}+\ldots . \\
& w=\tilde{w}^{(0)}+E^{1 / 12} \widetilde{w}^{(1)}+E^{2 / 12} \widetilde{w}^{(2)}+E^{3 / 12} \widetilde{w}^{(3)}+\ldots . \\
& p=E^{4 / 12} \tilde{p}^{(0)}+E^{5 / 12} \hat{p}^{(1)}+E^{6 / 12} \hat{p}^{(2)}+E^{7 / 12} \hat{p}^{(3)}+\ldots
\end{aligned}
$$

and substitution in the basic equations (2.4) results in

$$
\begin{aligned}
& 2 \hat{\mathcal{v}}^{(\mathrm{n})}=\frac{\partial \hat{p}^{(n)}}{\partial \eta}, \\
& 2 \hat{u}^{(n)}=\frac{\partial^{2} \widetilde{v}^{(n)}}{\partial \eta^{2}}, \\
& \frac{\partial \hat{p}^{(n)}}{\partial z}=\frac{\partial^{2} \mathfrak{w}^{(n)}}{\partial \eta^{2}}, \\
& \frac{\partial \tilde{u}^{(n)}}{\partial \eta}+\frac{\partial \widetilde{w}^{(n)}}{\partial z}=0,
\end{aligned}
$$

for $n=0,1,2,3$, where $\eta=(r-a) E^{-1 / 3}$ is the $1 / 3$-layer coordinate. Combination of (4.10a) (4.10d) leads to

$$
\left(\frac{\partial^{6}}{\partial \eta^{6}}+4 \frac{\partial^{2}}{\partial z^{2}}\right)\left(\tilde{v}^{(n)}, \tilde{w}^{(n)}\right)=0 .
$$

These quations (4.11) must be solved using boundary conditions derived from the 1/4-1ayersolutions.

As $V^{(0)}(\xi)$ could be made continuous at $\xi=0, h<z<H$ and satisfying the no-slip condition for $\xi=0,0<z<h$, the $(n=0)$-velocity field in the 1/3-layer plays an unimportant, passive role. The $(n=1)$-field, however, is important in order to remove the discontinuity in $\partial V^{(0)} / \partial \xi$, since

$$
E^{\frac{1}{4}} \frac{\partial v}{\partial r} \sim \frac{\partial V^{(0)}}{\partial \xi} \sim \frac{\partial v^{(1)}}{\partial \eta}
$$


In fact this $(n=1)$-field gives the structure of the 1/3-layer. It can be concluded that the $(n=2)$ field is not needed, since $\partial^{2} V^{(0)} / \partial \xi^{2}$ is continuous at $\xi=0$. Another reason for the abscence of this $(n=2)$-field is the fact that the total vertical $O\left(E^{\frac{1}{2}}\right)$-transport in the Stewartson layer must be zero. This can be proved by considering the net $O\left(E^{\frac{1}{2}}\right)$ mass flow through a horizontal plane at arbitrary $z$, including contributions of both interiors $I_{1}, I_{2}$ and the Stewartson layers at $r=1$ and $r=a$. The $(n=3)$-velocity field will be necessary in order to match $W^{(0)}$ to the no-slip condition $W^{(0)}(\xi=0)=0$ on $0<z<h$, besides to remove the discontinuity in $W^{(0)}$ at $\xi=0$, $h<z<H$.

The $\widetilde{v}^{(1)}, \widetilde{w}^{(1)}$ )-solution on $h<z<H$.

In the free part $(h<z<H)$ of the 1/3-layer we divide the solution domain into two parts: $-\infty<\eta \leqslant 0$ and $0 \leqslant \eta<+\infty$. At $\eta= \pm 0$ we demand continuity for the velocities and their first derivatives. Because of the jump in $\partial V^{(0)} / \partial \xi$ the continuity conditions then become

$$
\begin{aligned}
& \widetilde{v}_{+}^{(1)}\left(\eta=0^{+}\right)=\widetilde{v}_{-}^{(1)}\left(\eta=0^{-}\right), \\
& \left.\frac{\partial \widetilde{v}_{+}^{(1)}}{\partial \eta}\right|_{\eta=0^{+}}=\left.\frac{\partial \widetilde{v}_{-}^{(1)}}{\partial \eta}\right|_{\eta=0^{-}}, \\
& \widetilde{w}_{+}^{(1)}\left(\eta=0^{+}\right)=\widetilde{w}_{-}^{(1)}(\eta=0),
\end{aligned}
$$

whereas

$$
\eta \rightarrow \pm \infty: \widetilde{\mathcal{V}}^{(1)} \rightarrow 0, \widetilde{w}^{(1)} \rightarrow 0
$$

It can be easily verified, that the Ekman suction condition at $z=H$ in this particular case takes the following form:

$$
\widetilde{w}^{(1)}(\eta, H)=0 \text {. }
$$

Again considering the total mass flow in the cylinder through a horizontal plane at arbitrary $z$, now for $O\left(E^{5 / 12}\right)$, the total vertical $O\left(E^{5 / 12}\right)$-transport in the Stewartson layer at $r=a$ appears to be zero; so another condition can be formulated:

$$
\int_{-\infty}^{+\infty} \widetilde{w}^{(1)}(\eta, z) d \eta=0
$$

It appears that the discontinuity in $+\partial \widetilde{v}^{(1)} / \partial \eta$ leads to singular behaviour of $\widetilde{w}^{(1)}(\eta, h)$. The combination of (4.15) and the continuity at $\eta=0$ of $\mathcal{v}^{(1)}(\eta, z)$ and its derivatives, except of course $\partial \widetilde{v}^{(1)} / \partial \eta$, yields

$$
\widetilde{w}^{(1)}(\eta, h)=C \delta^{\prime}(\eta) \text {. }
$$


The unknown strength $C$ of this singularity depends on the jump in $\partial \tilde{v}^{(1)} / \partial \eta$, and will be determined later. By transformation techniques or by separation of variables the solutions of (4.11) which satisfy the conditions $(4.12)-(4.15)$ are

$$
\begin{aligned}
& \widetilde{v}_{+}^{(1)}(\eta, z)=\frac{2 C}{3(H-h)} \sum_{n=1}^{\infty} \frac{(-1)^{n}}{\gamma_{n}} \cos \left(n \pi \frac{z-H}{H-h}\right)\left\{e^{-\gamma_{n} n^{\eta}}+e^{-\frac{1}{2} \gamma_{n} \eta}\left(\cos \alpha_{n}-\sqrt{3} \sin \alpha_{n}\right)\right\} \\
& \tilde{v}_{-}^{(1)}(\eta, z)=\frac{2 C}{3(H-h)} \sum_{n=1}^{\infty} \frac{(-1)^{n}}{\gamma_{n}} \cos \left(n \pi \frac{z-H}{H-h}\right)\left\{e^{\gamma_{n} \eta}+e^{\frac{1}{2} \gamma_{n} \eta}\left(\cos \alpha_{n}+\sqrt{3} \sin \alpha_{n}\right)\right\} \\
& \widetilde{w}_{+}^{(1)}(\eta, z)=\frac{2 C}{3(H-h)} \sum_{n=1}^{\infty} \frac{(-1)^{n}}{\gamma_{n}} \sin \left(n \pi \frac{z-H}{H-h}\right)\left\{-e^{n_{+}^{\eta}} e^{-\frac{1}{2} \gamma_{n} \eta}\left(\cos \alpha_{n}-\sqrt{3} \sin \alpha_{n}\right)\right\}, \\
& \widetilde{w}_{-}^{(1)}(\eta, z)=\frac{-2 C}{3(H-h)} \sum_{n=1}^{\infty} \frac{(-1)^{n}}{\gamma_{n}} \sin \left(n \pi \frac{z-H}{H-h}\right)\left\{-e^{\gamma_{n} \eta}+e^{\frac{1}{2} \gamma_{n} \eta}\left(\cos \alpha_{n}+\sqrt{3} \sin \alpha_{n}\right)\right\},
\end{aligned}
$$

where $\alpha_{n}=\frac{1}{2} \gamma_{n} \eta \sqrt{ } 3$ and $\gamma_{n}^{3}=2 n \pi /(H-h)$.

From (4.16a) we deduce

$$
\begin{array}{l|l}
\frac{\partial \tilde{\nu}_{+}^{(1)}}{\partial \eta} & \eta=0^{+}=\frac{-2 C}{(H-h)} \sum_{n=1}^{\infty}(-1)^{n} \cos \left(n \pi \frac{z-H}{H-h}\right), \\
\frac{\partial \hat{\nu}_{-}^{(1)}}{\partial \eta} & \eta=0^{-}=\frac{+2 C}{(H-h)} \sum_{n=1}^{\infty}(-1)^{n} \cos \left(n \pi \frac{z-H}{H-h}\right) .
\end{array}
$$

Using the property of Fourier series (see Lighthill [5]),

$$
\sum_{n=1}^{\infty}(-1)^{n} \cos \frac{n \pi x}{l}=-\frac{1}{2}+l \sum_{m=-\infty}^{m=+\infty} \delta\{x-(2 m-1) l\}
$$

the summation term in (4.17) can be written as

$$
\sum_{n=1}^{\infty}(-1)^{n} \cos \left(n \pi \frac{z-H}{H-h}\right)=-\frac{1}{2}+(H-h) \sum_{m=-\infty}^{+\infty} \delta\{(z-H)-(2 m-1)(H-h)\},
$$

which represents an infinite series of delta functions in the points $z= \pm 2 m(H-h)+h$. These singular points lie just outside the region $h<z<H^{\star}$; therefore we may write

$$
\sum_{n=1}^{\infty}(-1)^{n} \cos \left(n \pi \frac{z-H}{H-h}\right)=-\frac{1}{2} \quad(h<z<H) .
$$

* In fact the singularity $\delta(z-h)$ lies just on the edge $z=h$ of the interval $h<z<H$; the edge $z=h$, however, is shielded by a transition region $h^{-}<z<h^{+}$(see Sec. 5). 
The jump in $\partial \tilde{v}^{(1)} / \partial \eta$ follows from the opposite jump in $\partial V^{(0)} / \partial \xi$, which can be deduced from (4.6a):

$$
\left.\frac{\partial \tilde{v}_{+}^{(1)}}{\partial \eta}\right|_{\eta=0^{+}}=-\left.\frac{\partial \tilde{v}_{-}^{(1)}}{\partial \eta}\right|_{\eta=0^{-}}=-\frac{1}{2} a \sqrt{\frac{2}{H}},
$$

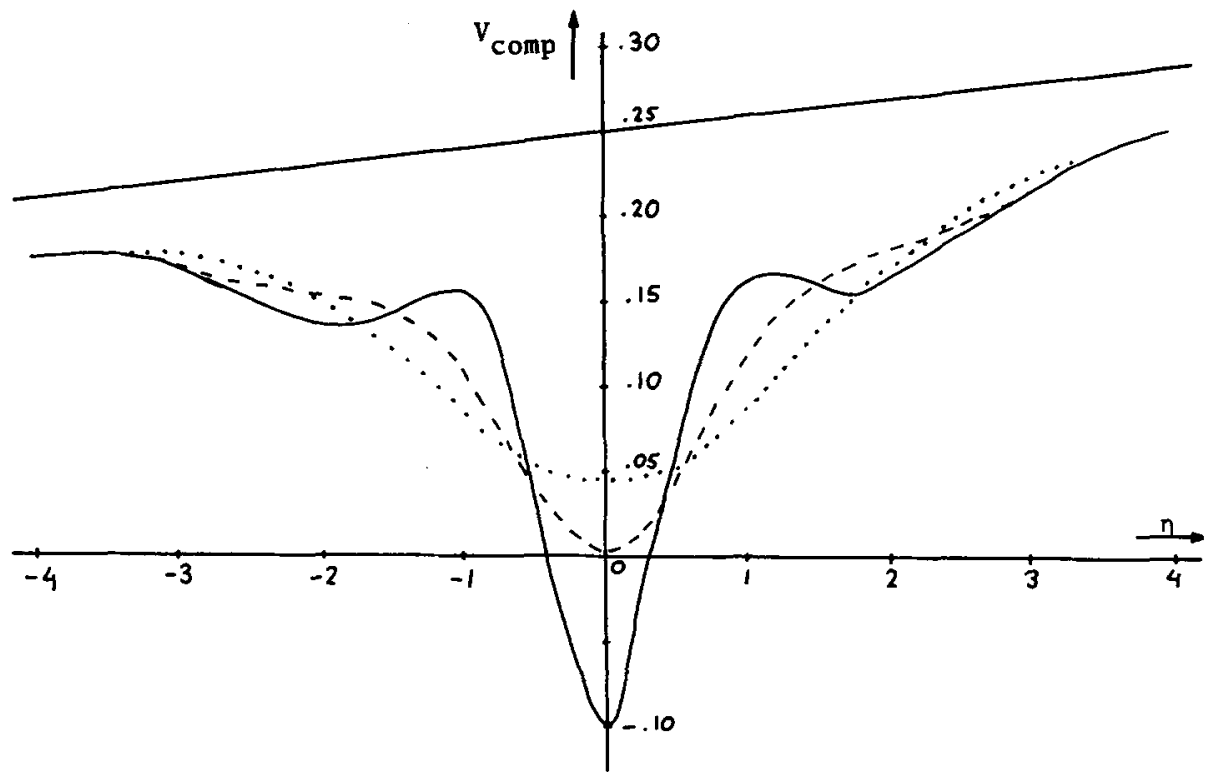

$z=0.3$

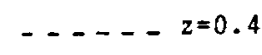

$\ldots \ldots \ldots=0.7$

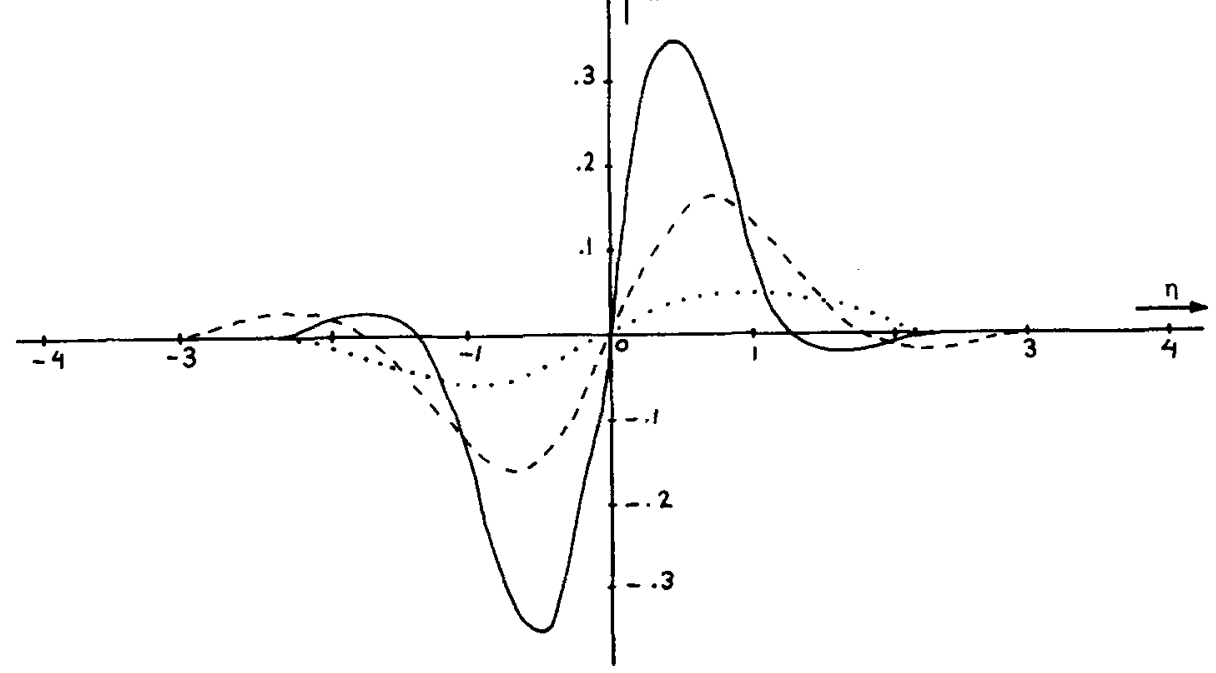

Figure 3. Velocity profiles of $V_{\text {comp }}=V^{(0)}+E^{1 / 12} \eta^{(1)}$ and $\mathcal{W}^{(1)}$ for $H=1, a=.5, h=.33, E=10^{-3}$. The straight solid line depicts the in terior velocity $v_{1}=\frac{1}{2} r=\frac{1}{2}\left(a+\pi E^{1 / 3}\right)$. 
and making use of (4.17), (4.18) we find

$$
C=-\frac{1}{2} a(H-h) \sqrt{\frac{2}{H}} .
$$

The strength $|C|$ of the singularity in $\widetilde{w}^{(1)}(\eta, h)$ appears to increase with increasing radius $a$, or decreasing strip height $h$.
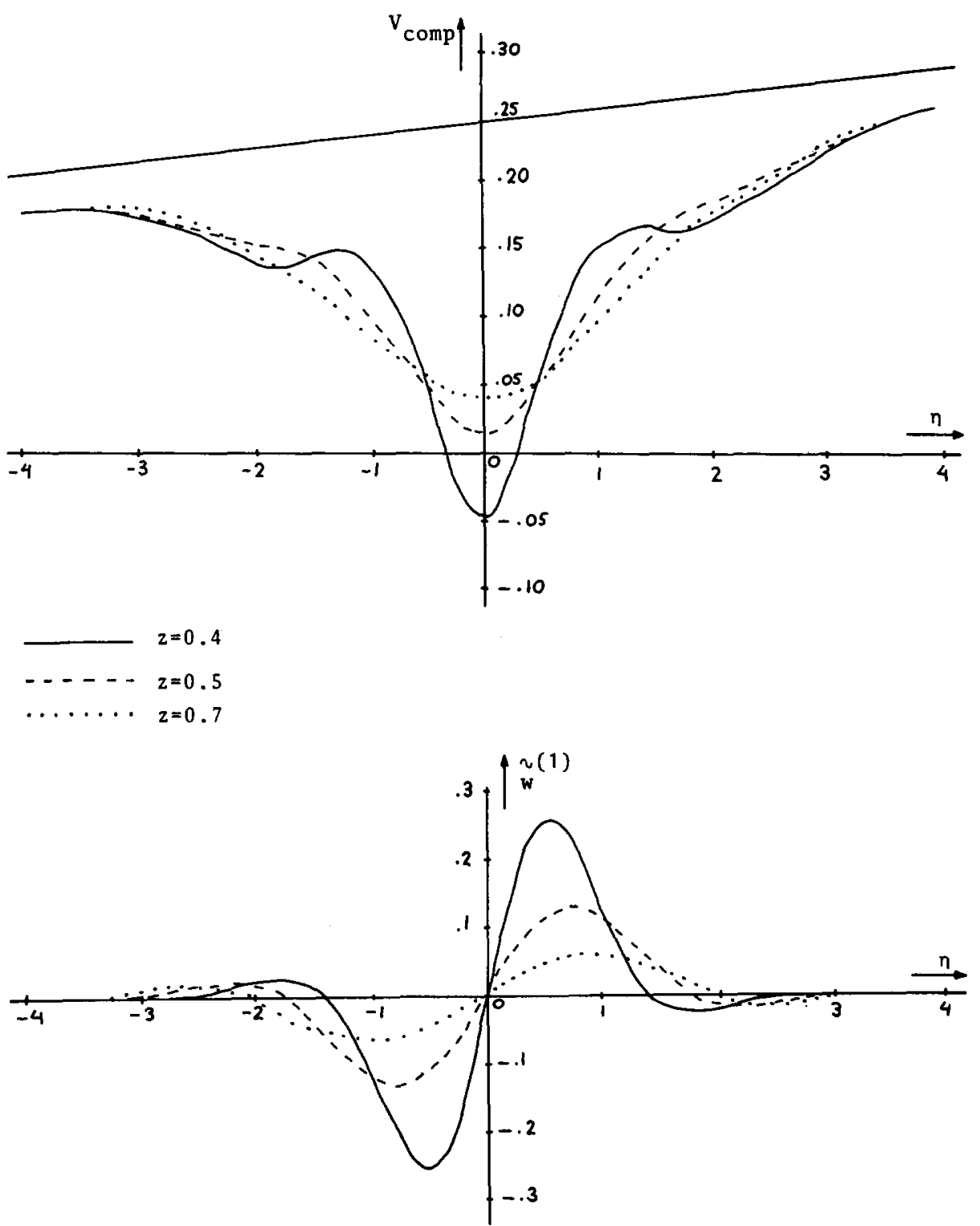

Figure 4. Velocity profiles of $V_{\text {comp }}=V^{(0)}+E^{1 / 12} \hat{q}^{(1)}$ and $\widetilde{w}^{(1)}$ for $H=1, a=.5, h=.25, E=10^{-5}$. The straight solid line depicts the interior velocity $v_{1}=\frac{1}{2} r=\frac{1}{2}\left(a+n E^{1 / 3}\right)$. 
Now the velocities $\widetilde{v}^{(1)}$ and $\widetilde{w}^{(1)}$ as given by (4.16) and (4.19) are fully determined. Figures 3 and 4 show velocity profiles of $V_{\text {com posite }}=V^{(0)}+E^{1 / 12} \widetilde{\nu}^{(1)}$ and $\widetilde{w}^{(1)}$ for different values of $z(>h)$.

The $\left(\tilde{v}^{(1)}, \tilde{w}^{(1)}\right.$-solution on $0<z<h$

Again we have to solve (4.11) in two separate regions, now subject to the following conditions:

$$
\begin{array}{ll}
\eta= \pm 0 & : \widetilde{v}^{(1)}=0, \widetilde{w}^{(1)}=0 \\
\eta \rightarrow \pm \infty & : \widetilde{v}^{(1)} \rightarrow 0, \widetilde{w}^{(1)} \rightarrow 0 ; \\
z=0 & : \widetilde{w}^{(1)}(\eta, 0)=0 \\
z=h & \widetilde{w}^{(1)}(\eta, h)=C \delta^{\prime}(\eta) \\
\int_{-\infty}^{0^{-}} \widetilde{w}_{-}^{(1)}(\eta, z) d \eta+\int_{0}^{+\infty} \widetilde{w}_{+}^{(1)}(\eta, z) d \eta=0
\end{array}
$$

Once again we assume $\widetilde{w}^{(1)}(\eta, h)$ to behave like $\delta^{\prime}(\eta)$, just as in the 1/3-layer part on $h<z<H$. There will occur an "intermediate region" at $h^{-}<z<h^{+}$, as will be shown later, but this "layer" is too thin, thickness of order $E^{1 / 2}$, to absorb the $\delta^{\prime}(\eta)$ singularity. We will return to this point in the next section. The procedure of solving (4.11) is straight forward, and results in:

$$
\begin{aligned}
& \widetilde{v}_{+}^{(1)}(\eta, z)=\frac{-4 C}{\pi \sqrt{3}} \sum_{n=1}^{\infty} \frac{(-1)^{n}}{n} \beta_{n} \cos \frac{n \pi z}{h} e^{-\frac{1}{2} \beta_{n} \eta} \sin \frac{1}{2} \beta_{n} \eta \sqrt{3} \\
& \widetilde{v}_{-}^{(1)}(\eta, z)=\frac{+4 C}{\pi \sqrt{3}} \sum_{n=1}^{\infty} \frac{(-1)^{n}}{n} \beta_{n} \cos \frac{n \pi z}{h} e^{\frac{1}{2} \beta_{n} \eta} \sin \frac{1}{2} \beta_{n} \eta \sqrt{3} \\
& \widetilde{w}_{+}^{(1)}(\eta, z)=\frac{4 C}{\pi \sqrt{3}} \sum_{n=1}^{\infty} \frac{(-1)^{n}}{n} \beta_{n} \sin \frac{n \pi z}{h} e^{-\frac{1}{2} \beta_{n} \eta} \sin \frac{1}{2} \beta_{n} \eta \sqrt{3} \\
& \widetilde{w}_{-}^{(1)}(\eta, z)=\frac{4 C}{\pi \sqrt{3}} \sum_{n=1}^{\infty} \frac{(-1)^{n}}{h} \beta_{n} \sin \frac{n \pi z}{h} e^{\frac{1}{2} \beta_{n} \eta} \sin \frac{1}{2} \beta_{n} \eta \sqrt{3}
\end{aligned}
$$

where $\beta_{n}^{3}=2 n \pi / h$ and $C$ are given by (4.19).

Considering the solutions (4.16) and (4.23) for the free resp. attached parts of the 1/3-layer, it appears that both tangential velocities $\widetilde{v}^{(1)}$ do not match smoothly at $z=h$. The coupling of both $\widetilde{\mathcal{v}}^{(1)}$-solutions has to take place in a thin layer, an "intermediate domain", in which derivatives in axial direction are assumed to be considerable. This will be worked out in Sec. 5 .

The $\left(\tilde{v}^{(3)}, \widetilde{w}^{(3)}\right)$-solution on $0<z<H$.

This $(n=3)$-velocity field of the $1 / 3$-layer exists in order to remove the discontinuity in $W^{(0)}$ 
on $\xi=0, h<z<H$ and to match $W^{(0)}(\xi=0)$ to zero on the rigid strip surface $\eta=0,0<z<h$. Since $V^{(1)}$ and $\tilde{v}^{(3)}$ are of the same order of magnitude, $\widetilde{v}^{(3)}$ possibly has to match $V^{(1)}(\xi=0)$, as given by (4.9), to zero at $\eta=0,0<z<h$. Another condition follows from the fact that the total vertical $O\left(E^{7 / 12}\right)$-transport in this Stewartson layer has to be zero. This can be shown by considering the total vertical transport of $O\left(E^{7 / 12}\right)$ in the cylinder at arbitrary $z$, including contributions of both interiors and the Stewartson layers at $r=1$ and $r=a$. Equation (4.11) has to be solved subject to some boundary conditions, which we can formulate as:

$$
\begin{aligned}
& \eta=0,0<z<h: \widetilde{\mathcal{V}}_{+}^{(3)}\left(\eta=0^{+}\right)+V_{+}^{(1)}\left(\xi=0^{+}\right)=\widetilde{v}_{-}^{(3)}\left(\eta=0^{-}\right)+V_{-}^{(1)}\left(\xi=0^{-}\right)=0, \\
& \eta=0,0<z<h: \widetilde{w}_{+}^{(3)}\left(\eta=0^{+}\right)+W_{+}^{(0)}\left(\xi=0^{+}\right)=\widetilde{w}_{-}^{(3)}\left(\eta=0^{-}\right)+W_{-}^{(0)}\left(\xi=0^{+}\right)=0, \\
& \eta=0, h<z<H: \widetilde{w}_{+}^{(3)}\left(\eta=0^{+}\right)+W_{+}^{(0)}\left(\xi=0^{+}\right)=\widetilde{w}_{-}^{(3)}\left(\eta=0^{-}\right)+W_{-}^{(0)}\left(\xi=0^{+}\right), \\
& z=0 \text { and } z=H: \widetilde{w}^{(3)}(\eta, 0)=\widetilde{w}^{(3)}(\eta, H)=0, \\
& 0<z<H: \int_{-\infty}^{0-} \widetilde{w}_{-}^{(3)}(\eta, z) d \eta+\int_{0}^{+} \widetilde{w}_{+}^{(3)}(\eta, z) d \eta=0 .
\end{aligned}
$$

Boundary condition (4.25) expresses the Ekman suction condition at the boundaries of both Ekman layers.

Following the same procedure as before, we derive for the velocity components:

$$
\begin{aligned}
& \widetilde{v}_{+}^{(3)}(\eta, z)=\sum_{n=1}^{\infty} a_{n} \cos \frac{n \pi z}{H}\left\{e^{-\gamma_{n} \eta}-e^{-\frac{1}{2} \gamma_{n} \eta}\left(\cos \alpha_{n}-\sqrt{3} \sin \alpha_{n}\right)\right\}, \\
& \widetilde{v}_{-}^{(3)}(\eta, z)=\sum_{n=1}^{\infty} a_{n} \cos \frac{n \pi z}{H}\left\{e^{\gamma_{n} \eta}-e^{\frac{1}{2} \gamma_{n} \eta}\left(\cos \alpha_{n}-\sqrt{3} \sin \alpha_{n}\right)\right\} \\
& \widetilde{w}_{ \pm}^{(3)}(\eta, z)=\sum_{n=1}^{\infty} a_{n} \sin \frac{n \pi z}{H}\left\{e^{-\gamma_{n} \eta}+e^{-\frac{1}{2} \gamma_{n} \eta}\left(\cos \alpha_{n}-\sqrt{3} \sin \alpha_{n}\right)\right\}, \\
& \widetilde{w}_{-}^{(3)}(\eta, z)=\sum_{n=1}^{\infty} a_{n} \sin \frac{n \pi z}{H}\left\{e^{\gamma_{n} \eta}+e^{\frac{1}{2} \gamma_{n} \eta}\left(\cos \alpha_{n}+\sqrt{3} \sin \alpha_{n}\right)\right\},
\end{aligned}
$$

where

$$
a_{n}=-\frac{a}{4 \pi} \sqrt{\frac{2}{H}} \frac{(-1)^{n}+1}{n}, \quad \alpha_{n}=\frac{1}{2} \gamma_{n} \eta \sqrt{3}, \quad \gamma_{n}^{3}=2 n \pi / H
$$

One can verify that

$$
\begin{aligned}
\widetilde{w}_{ \pm}^{(3)}(\eta=0) & =\mp \frac{a}{2 \pi} \sqrt{\frac{2}{H}} \sum_{n=1}^{\infty} \frac{(-1)^{n}+1}{n} \sin \frac{n \pi z}{H} \\
& =\mp \frac{a}{2 \pi} \sqrt{\frac{2}{H}} \sum_{n=1}^{\infty} \frac{1}{n} \sin \frac{2 n \pi z}{H}=\mp \frac{a}{2} \sqrt{\frac{2}{H}}\left(\frac{1}{2}-\frac{z}{H}\right)
\end{aligned}
$$


so (4.27b) obeys (4.24c). It is easily shown that the other boundary conditions (4.24a) and (4.24b) are satisfied as well, giving $V_{+}^{(1)}\left(\xi=0^{+}\right)=V_{-}^{(1)}\left(\xi=0^{-}\right)=0$.

The $\left(\tilde{\mathcal{V}}^{(3)}, \widetilde{w}^{(3)}\right)$-solution appears to be independent of the strip height $h$; in other words, the (no-slip) conditions (4.24a) and (4.24b) are also satisfied in $h<z<H$. So the velocities (4.27) are not affected by the strip height, and no singularity at $z=h$ is introduced in the $(n=3)$ field.

\section{The "quasi-Ekman layer"}

As stated before in the 1/3-layer analysis, an intermediate region is necessary to match both tangential 1/3-layer velocity components $\widetilde{v}^{(1)}$ at $z=h^{-}$and $z=h^{+}$. Since $\widetilde{v}^{(1)}(\eta \rightarrow \pm \infty) \rightarrow 0$, the intermediate layer only exists for $|a-r| \sim E^{1 / 3}$, and fades away for $\eta \rightarrow \pm \infty$.

Assuming the axial dimension to be small, derivatives in axial direction may be considerable in order to accomplish the coupling of the 1/3-layer solutions. The intermediate domain is then defined by

$$
|z-h|<E^{1 / 2}, \quad E^{1 / 3}<|a-r|<E^{5 / 12}
$$

because, as we will show by transport considerations later in this section, at the strip edge a singular region of dimensions $E^{1 / 2} \times E^{5 / 12}$ must exist (see Figure 5).

Now we introduce a set of characteristic coordinates

$$
\zeta=(z-h) E^{-\frac{1}{2}}, \quad \eta=(r-a) E^{-1 / 3},
$$

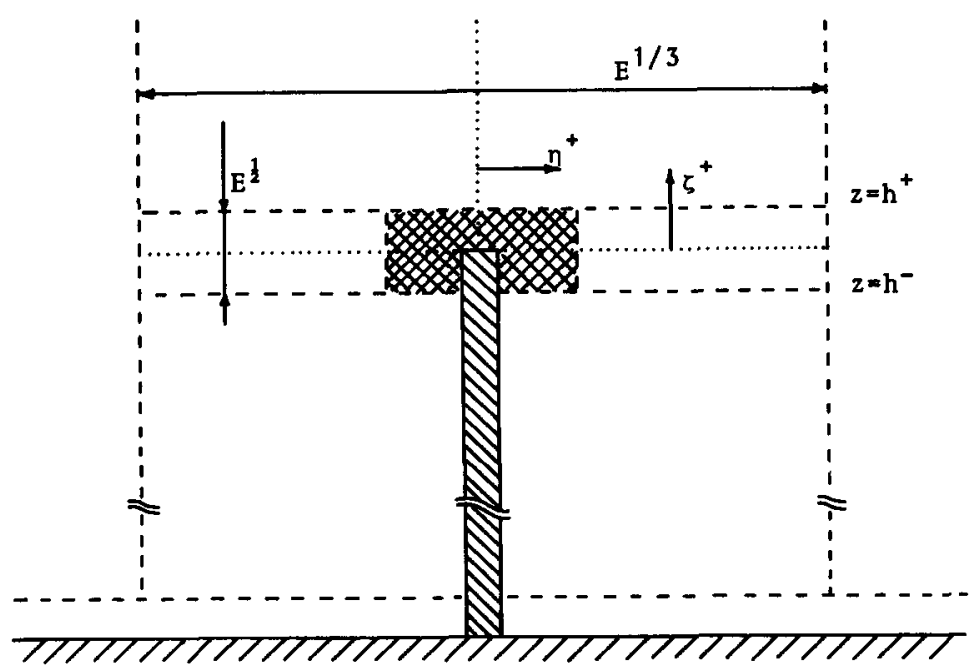

Figure 5. Structure of the quasi-Ekman layer at $z=h$. The shaded section indicates the singular region. 
and expand the velocity components and pressure into the following perturbation series:

$$
\begin{aligned}
& u=E^{1 / 12} \bar{u}^{(1)}+E^{2 / 12} \bar{u}^{(2)}+\ldots, \\
& v=E^{1 / 12} \bar{\nu}^{(1)}+E^{2 / 12} \bar{\nu}^{(2)}+\ldots, \\
& w=E^{3 / 12} \bar{w}^{(1)}+E^{4 / 12} \bar{w}^{(2)}+\ldots, \\
& p=E^{1 / 12} \bar{p}^{(1)}+E^{2 / 12} \bar{p}^{(2)}+\ldots
\end{aligned}
$$

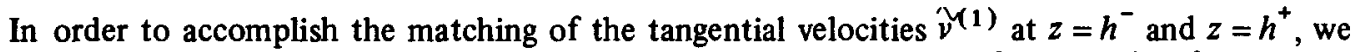
choose the leading term in the vexpansion as being of $O\left(E^{1 / 12}\right)$. Since $\widetilde{w}^{(1)}\left(\eta, h^{+}\right)=\widetilde{w}^{(1)}\left(\eta, h^{-}\right)$ $=0$ for $\eta \neq 0$, owing to the $\delta^{\prime}(\eta)-$ like behaviour of $\hat{w}^{(1)}(\eta, h)$, there is no need for choosing $\bar{w}^{(1)} \sim O\left(E^{1 / 12}\right)$. Substitution of the expansions (5.3) into the basic equations (2.4) yields

$$
\begin{aligned}
& -2 \bar{\nu}^{(j)} / \partial \zeta=\partial^{3} \bar{u}^{(j)} / \partial \zeta^{3}, \\
& 2 \bar{u}^{(j)}=\partial^{2} \bar{v}^{(j)} / \partial \zeta^{2}, \\
& \partial \bar{p}^{(j)} / \partial \zeta=0 \\
& \partial \bar{u}^{(j)} / \partial \eta+\partial \bar{w}^{(j)} / \partial \zeta=0,
\end{aligned}
$$

for $j=1,2$. Because of the strong analogy between these expressions and the governing equations (3.4) of the usual Ekman layer, the intermediate layer will be referred to as "quasi-Ekman layer".

The solution of these equations (5.4) is standard and yields

$$
\begin{aligned}
& \bar{u}_{+}^{(1)}=a_{1}(\eta) e^{-(1+i) \zeta}+a_{2}(\eta) e^{-(1-i) \xi}, \\
& \bar{u}_{-}^{(1)}=a_{3}(\eta) e^{(1+i) \zeta}+a_{4}(\eta) e^{(1-i) \zeta} ; \\
& \bar{v}_{+}^{(1)}=-i a_{1}(\eta) e^{-(1+i) \zeta}+i a_{2}(\eta) e^{-(1-i) \zeta}+\vartheta^{(1)}\left(\eta, h^{+}\right) \\
& \bar{v}_{-}^{(1)}=-i a_{3}(\eta) e^{(1+i) \zeta}+i a_{4}(\eta) e^{(1-i) \zeta}+\tilde{v}^{(1)}\left(\eta, h^{-}\right),
\end{aligned}
$$

where the indices \pm denote the cases $\zeta \geqslant 0$ resp. $\zeta \leqslant 0$. Requiring continuity of $\bar{u}^{(1)}, \bar{v}^{(1)}$ and their $\zeta$-derivatives at $\zeta=0$ determines the integration "constants" $a_{m}(\eta)$, resulting in

$$
\begin{aligned}
& \bar{u}_{+}^{(1)}(\eta, \zeta)=-f(\eta) e^{-\zeta} \sin \zeta \\
& \bar{u}_{-}^{(1)}(\eta, \zeta)=-f(\eta) e^{\zeta} \sin \zeta \\
& \bar{v}_{+}^{(1)}(\eta, \zeta)=-f(\eta) e^{-\zeta} \cos \zeta+\widetilde{v}^{(1)}\left(\eta, h^{+}\right) \\
& \bar{v}_{-}^{(1)}(\eta, \zeta)=f(\eta) e^{\zeta} \cos \zeta+\widetilde{v}^{(1)}\left(\eta, h^{-}\right)
\end{aligned}
$$

with

$$
f(\eta)=\frac{1}{2}\left\{\widetilde{v}^{(1)}\left(\eta, h^{+}\right)-\widetilde{v}^{(1)}\left(\eta, h^{-}\right)\right\}
$$


As we have seen before, a vertical $O\left(E^{5 / 12}\right)$-transport takes place in the $\left(\widetilde{\nu}^{(1)}, \widetilde{w}^{(1)}\right)$-field of the 1/3-layer. However, according to the expansions (5.3), the transport-integral

$$
\int_{0}^{\infty} \bar{w}^{(j)} d \eta
$$

never can be of $O\left(E^{5 / 12}\right)$. This means that the $O\left(E^{5 / 12}\right)$-transport between both 1/3-layer parts $\left(0<z<h^{-}\right.$and $\left.h^{+}<z<H\right)$ has to take place in a singular region at $\eta=0$. The existence of this singularity is directly connected with the $\delta^{\prime}(\eta)$-like behaviour of $\widetilde{w}^{(1)}(\eta, h)$.

In the singular region we assume $\hat{w}=\hat{w}^{(1)}+\ldots$ and demand

$$
\int \hat{w} d \rho=O\left(E^{5 / 12}\right)
$$

where $\rho$ is the characteristic lateral coordinate. The only possibility is that $\rho \sim O\left(E^{5 / 12}\right)$, so that the singularity occurs in a region of dimensions $E^{1 / 2} \times E^{5 / 12}$. The smooth out this singularity in $z$ direction would require a $\partial w / \partial z$ of order $E^{-5 / 12}$. This must be balanced by $\partial u / \partial r$ and because $\partial / \partial r \sim E^{-5 / 12}$ in the singularity region, radial velocities of unit order would be produced. These are much larger than the radial velocities in the surrounding $1 / 3$-layer and in the interior. No large radial velocities are required if we rule out the smoothing of the singularity in $z$-direction and assume that, like the $\widetilde{w}^{(1)}$-field in $h<z<H$, also the $\widetilde{w}^{(1)}$-field in $0<z$ $<h$ behaves like $\delta^{\prime}(\eta)$ when $z$ approaches $h . *$

In the previous section we calculated the $\left(\widetilde{v}^{(3)}, \widetilde{w}^{(3)}\right)$ - velocity field in the 1/3-layer, which tumed out to be independent of the strip height $h$. In the quasi-Ekman layer the vertical velocity $\bar{w}^{(1)}$ is of $O\left(E^{3 / 12}\right)$, so is of the same order of magnitude as $\widetilde{w}^{(3)}$. Now we wonder whether this intermediate layer is affecting the $h$-independent $\widetilde{w}^{(3)}$-solution in the 1/3-layer. Integrating (5.4d) successively with respect to $\zeta$ and $\eta$ we obtain

$$
\begin{aligned}
& \int_{-\infty}^{0-}\left(\int_{-\infty}^{+\infty} \frac{\partial \bar{u}^{(1)}}{\partial \eta} d \zeta\right) d \eta+\int_{0^{+}}^{+\infty}\left(\int_{-\infty}^{+\infty} \frac{\partial \bar{u}^{(1)}}{\partial \eta} d \zeta\right) d \eta+ \\
& +\int_{-\infty}^{+\infty} \bar{w}^{(1)}(\zeta=+\infty) d \eta-\int_{-\infty}^{+\infty} \bar{w}^{(1)}(\zeta=-\infty) d \eta=0
\end{aligned}
$$

and using (5.6), (4.16) and (4.23) this can be written as

$$
\int_{-\infty}^{+\infty} \bar{w}^{(1)}(\zeta=+\infty) d \eta=\int_{-\infty}^{+\infty} \bar{w}^{(1)}(\zeta=-\infty) d \eta
$$

Since

$$
\begin{aligned}
& \lim _{\zeta \rightarrow+\infty} \bar{w}^{(1)}(\eta, \zeta)=\widetilde{w}^{(3)}\left(\eta, h^{+}\right), \\
& \lim _{\zeta \rightarrow-\infty} \bar{w}^{(1)}(\eta, \zeta)=\widetilde{w}^{(3)}\left(\eta, h^{-}\right),
\end{aligned}
$$

* This assumption is similar to adopting a sort of Kutta condition like in Moore \& Saffman [6]. 
(5.9) expresses the fact that the vertical $O\left(E^{7 / 12}\right)$-transport in the 1/3-layer is not affected by the presence of the quasi-Ekman layer at $h^{-}<z<h^{+}$.

\section{Conclusion}

As we have seen, due to the presence of a thin strip of small height fixed upon the bottom disk, a Stewartson layer occurs that is partially free, partially attached. The 1/4-layer appears to be independent of the strip height $h$, owing to its quasi-geostrophic character. The 1/3-layer problem, however, has to be solved in two separate intervals $0<z<h$ and $h<z<H$, in order to satisfy the no-slip condition at the strip surface. Since the $1 / 3$-layer velocities $\tilde{v}^{(1)}$ do not match at $z=h$, an intermediate layer had to be introduced. The structure of this intermediate region turns out to be analogous to the usual Ekman-layer structure. The difference lies in the fact that this quasi-Ekman layer is free, in contrast with a usual Ekman layer, which is always attached to some solid horizontal boundary.

\section{Acknowledgement}

The author is very much indebted to Professor L. van Wijngaarden for many illuminating discussions concerning the subject of this paper.

\section{REFERENCES}

[1] D. L. Boyer, Rotating flow over a step, J. Fluid Mech. 50 (1971) 675-687.

[2] M. R. Foster, The flow caused by the differential rotation of a right circular cylindrical depression in one of two rapidly rotating parallel planes, J. Fluid Mech. 53 (1972) 647-655.

[3] M. R. Foster, Rotating flow past an elliptic-cylindrical bump of large aspect ratio, J. Appl. Math. Phys. 26 (1975) 789-806.

[4] H. P. Greenspan, The theory of rotating fluids, Cambridge University Press (1968).

[5] M. J. Lighthill, Introduction to Fourier-analysis and generalised functions, Cambridge University Press (1958).

[6] D. W. Moore \& P. G. Saffman, The structure of free vertical shear layers in a rotating fluid and the motion produced by a slowly rising body, Phil. Trans. Roy. Soc. Lond. A264 (1969) 597-634.

[7] K. Stewartson, On almost rigid rotations, J. Fluid Mech. 3 (1957) 17-26.

[8] K. Stewartson, On almost rigid rotations, Part 2, J. Fluid Mech. 26 (1966) 131-144. 